\title{
On-line observation of interlaminar damage by ultrasonic inspection
}

\author{
Yanjin Dong*, Ning Ye, Yilong Bai \\ Laboratory for Nonlinear Mechanics of Continuous Media, Institute of Mechanics, Chinese Academy of Sciences, Beijing 100080, People's Republic of China
}

Received 6 March 1998; received in revised form 3 June 1998; accepted 30 July 1998

\begin{abstract}
This paper explores an on-line experimental method to highlight the process of internal damage development in composites by taking advantage of ultrasonic inspection. A loading device, which can work together with an ultrasonic inspection system, was designed, and the interlaminar shear damage of a double-sided grooved specimen of composite was examined on-line with the system. A full view of the progressive internal interlaminar damage, seen only with difficulty by common inspection methods, was successfully achieved. (C) 1999 Elsevier Science Ltd. All rights reserved.
\end{abstract}

Keywords: A. Polymer-matrix composites; C. Crack; D. Ultrasonics; Damage

\section{Introduction}

Experimental techniques are paramount in composite mesomechanics. They aid us in better formulating the basic laws of mechanics. Theoretical analysis and numerical simulation are soundly reliable only when they are set up on an experimental basis. Experimental observations highlight not only novel phenomena, but also their underlying mechanisms. The experimental techniques of mesomechanics are now progressing rapidly and have been turning into a typical interdiscipline, with the help of new accomplishments and progress of various disciplines [1,2].

In situ tests and on-line experiments are two important factors mostly expected in the mesomechanics research. The conventional methods used to observe the deformation process are the multi-specimen method or the singlespecimen method. In the former the specimens are loaded to different deformation stages, while in the latter one specimen is loaded and unloaded repeatedly many times in order to record the deformation of the specimen at different stages. However, neither of these can provide us with a realistic and accurate recording of the deformation development of a sample under load. In situ and on-line tests have enjoyed growing favor among researchers. For example, the deformation evolution on the specimen surface has been monitored by using SEM with a loading stage or a Questar remote measurement system equipped with an MTS material testing system in

\footnotetext{
* Corresponding author.
}

our laboratory. Moreover, the deformation of solid materials can be measured by holography, speckle-pattern interferometry $[3,4]$ or other techniques. However, with all of these methods it is impossible to detect the defects and damage growth processes inside solid materials. As is well known, it is extremely important to understand how defects generate and grow inside a material under load. Techniques such as $\mathrm{x}$-radiography and ultrasonic wave methods which can detect the inside of materials are therefore usually chosen to fulfill the task. The distinguishing feature of ultrasonic inspection is that ultrasonic pulses can easily penetrate solid objects and detect internal flaws in materials [5-7]. In addition, this method is harmless to users. Researchers are therefore increasingly turning to ultrasonic inspection for revealing the deformation mechanisms of materials $[8,9]$. In this paper, an ultrasonic inspection system is adopted to explore an on-line experimental method in order to highlight the process of internal damage development in composites by taking advantage of ultrasonic inspection. Actually, we expect to reveal the internal damage evolution in a woven glass-fabric composite by using the immersion ultrasonic C-scanning technique.

\section{Experiment}

\subsection{Test specimen}

The interlaminar shear damage which occurs in a double-sided grooved specimen is the delamination 
between laminae in the middle of the specimen. Usually, observation of delaminations in composites is made either by microscope or by ultrasonic C-scanning system after testing. In order to examine the details of the extension of interlaminar damage, the specimen must be cut in two. But, in this way, we may only observe one stage of the damage growth process. However, the damage extension which develops with increasing load or deformation is more worth investigating. The ultrasonic inspection is therefore, adopted to examine the damage growth process of a composite in an interlaminar shear test. C-scanning seems perfect for the study of interlaminar damage in the double-sided grooved composite specimen for the following reasons:

(1) The surface of specimen is even. This guarantees that almost no deflection of ultrasonic pulses can occur on the specimen;

(2) The cracks do not overlap each other. The cracks in deeper positions cannot be found if they overlap each other along the transmission direction;

(3) The crack propagates evenly and straight, which guarantees that the echo can return to the probe along the original transmission direction. The interlaminar shear test of double-sided grooved specimen was therefore, performed with the on-line experimental technique by using the USIP 20 ultrasonic C-scanning test system.

The test material is a woven glass-fabric-reinforced epoxy composite. The plain-weave glass fabrics contained 44 (warp) $\times 34$ (weft) strands per $2.5 \mathrm{~cm} \times 2.5 \mathrm{~cm}$ square area. Each strand consists of 400 filaments, $9 \mu \mathrm{m}$ in diameter. The configuration and dimension of the specimen is given in Fig. 1.

\subsection{Loading device}

A loading device equipped with $\mathrm{C}$-scanning water tank was designed and constructed in order to highlight the damage development processes at different loading
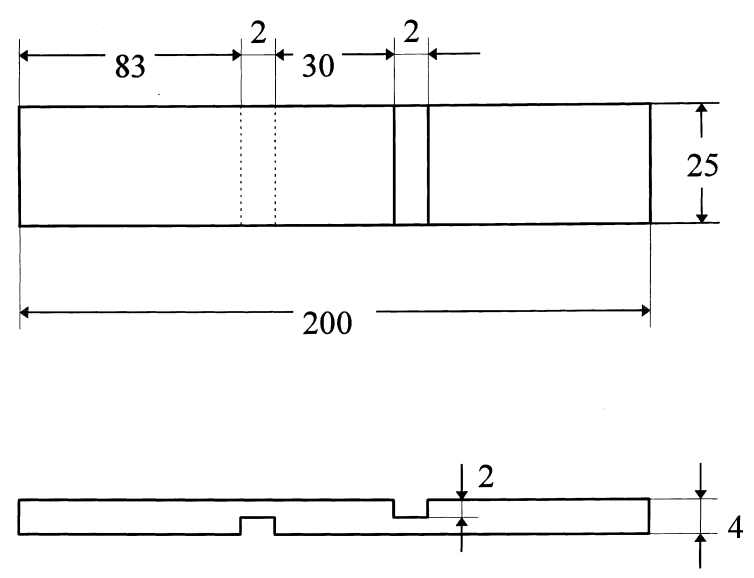

Fig. 1. The double-sided grooved specimen. stages. The loading device can work together with the USIP 20 ultrasonic C-scanning test system. The design of the loading device in this paper is mainly aimed at the interlaminar shear testing of composites.

There are many factors to be taken into account in the design of the loading device. Firstly, both maximum load and maximum displacement were determined by the tests performed on an Instron materials testing machine. The next stage was to work out how to load the sample in the water tank of the C-scanning system. A schematic illustration of the loading device is shown in Fig. 2. The left part is a decelerator with a handle. When the test was performed, the handle was turned round and round. The load was transferred from the handle to the decelerator, which was used to reduce the loading velocity and change the loading direction. Then, the working stage of the flat nosed pliers was slid along the sliding track with the decelerator. The load was transferred from the decelerator to the flat-nosed pliers, then to the load arm and finally to the specimen $[10,11]$. After that, the grips were designed to satisfy the following three requirements: (1) the load should be acted along the longitudinal axis of specimen; (2) the assembly and disassembly should be very easy; (3) the specimen should not slip from the grips during the loading process.

In addition, the device was limited by the size of the water tank and the scanning system, see Fig. 2.

\subsection{On-line experiments}

The interlaminar shear tests were performed with the loading device. The test was displacement controlled manually. A digital dial indicator fixed up with the pliers indicated the total displacement of specimen. The ultrasonic C-scanning tests were performed under an immersion type pulse-echo mode utilizing a $5 \mathrm{MHz}$ focused transducer. Reflections from the front surfaces of the specimen (RFSS) and a glass plate (RGP) under the specimen were all captured by the ultrasonic equipment monitor and then gated. The method is usually used to detect thin plates. The deformed specimens were scanned at different loading stages.

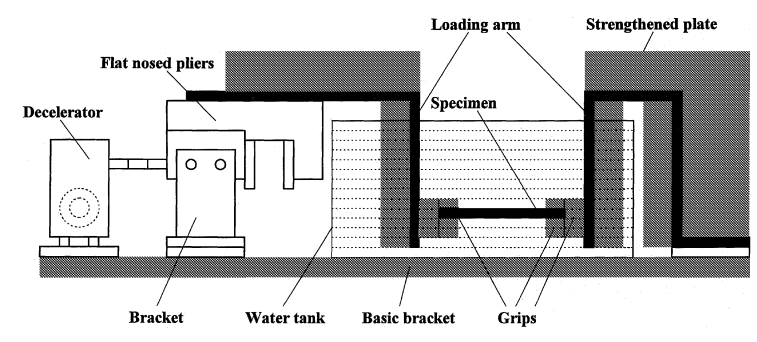

Fig. 2. A schematic illustration of the loading device. 


\section{Results and discussion}

Fig. 3(a) shows the C-scanning image obtained before loading. From the figure, one can easily see the outline of specimen and the two grooves. Fig. 3(b)-(d) show the C-scanning images obtained during the loading process and they correspond to different deformations respectively. The last three images in Fig. 3 are zoomed for clarity. During the scanning process, the interface echo near the left groove is not disturbed by the distortion of the groove edge because the left groove locates on the back of specimen. So we focus our attention to the image of the interlaminar crack and damage near the left groove.

The above images consist of 18 colors. Different color demonstrates different amplitude of pulse echo. The RGP amplitude ranges from $0.0 \%$ to $6.4 \%$ full screen deflection (FSD) with the transit of black to white. The increment of amplitude is $0.4 \%$ FSD. The color scale is shown at the right-upper corner of the image. During the on-line test, it is ensured that the colors were identically defined for all images, provided both minimum and maximum of RGP amplitude are unchanged in all images.

The edge of the specimen is determined by the halfwave method [12]. The basis of the method is derived from the quantitative determination of shape and size of flaws in ultrasonic inspection area. At first, the maximum RGP amplitude of the intact region is measured. A border region whose amplitude equals half the maximum is then defined as the edge of the specimen. In Fig. 3, the maximum amplitude is $6.4 \%$ FSD. A half of the amplitude is $3.2 \%$ FSD. From Fig. 3(a), the half of the maximum RGP almost locates at the middle of the transitional region of colours. Clearly, this does indicate the boundary of the specimen. When the RGP amplitude of a region is lower than a half of the maximum RGP, the region is then considered as cracked. Concretely, the boundary between $2.8 \%$ FSD and $3.2 \%$ FSD (around the middle of the green region) is taken as the front of the interlaminar crack. The zoom of the crack front is shown at the right-botton corner in Fig. 3(d). The crack front can be easily distinguished in the zoom with the enhanced edge. We can now a

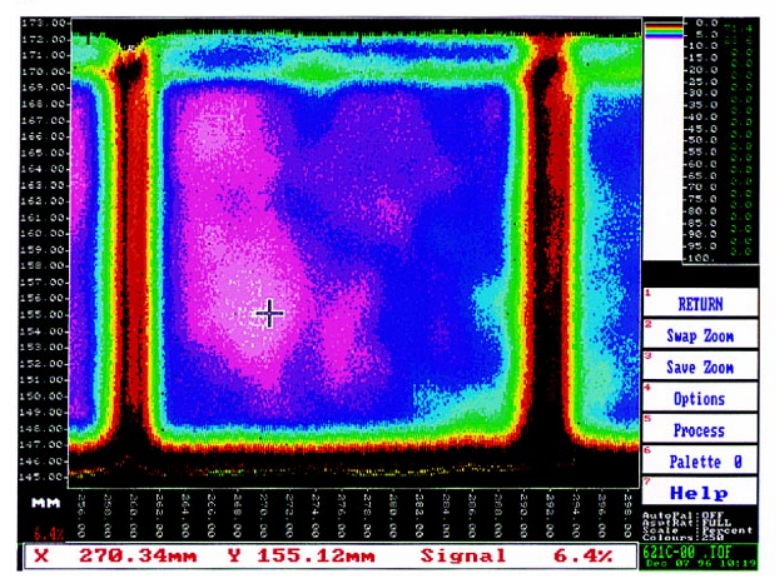

C

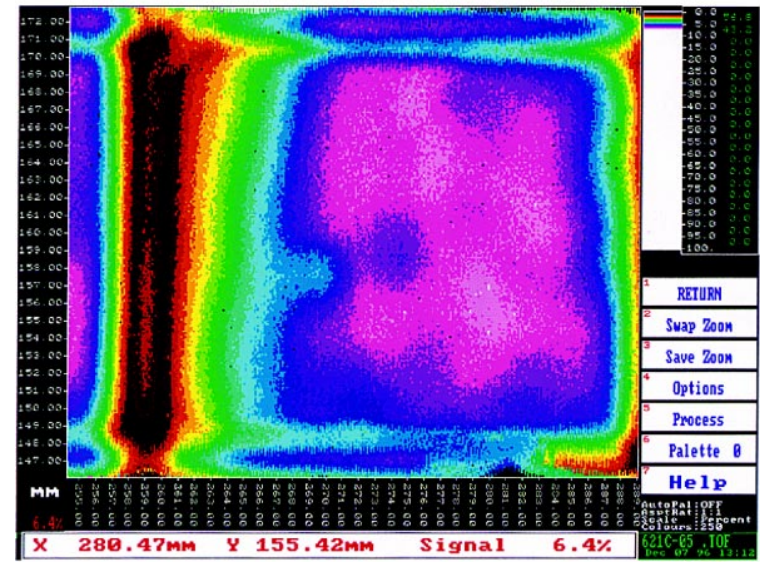

b

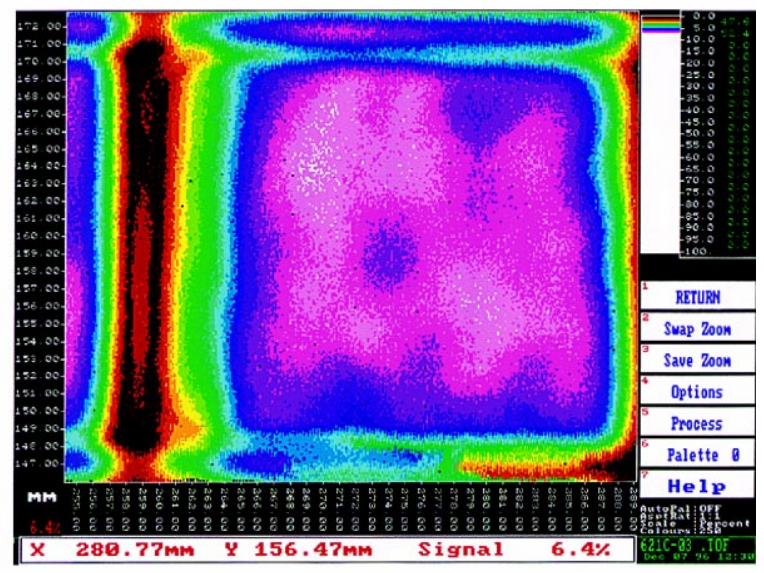

d

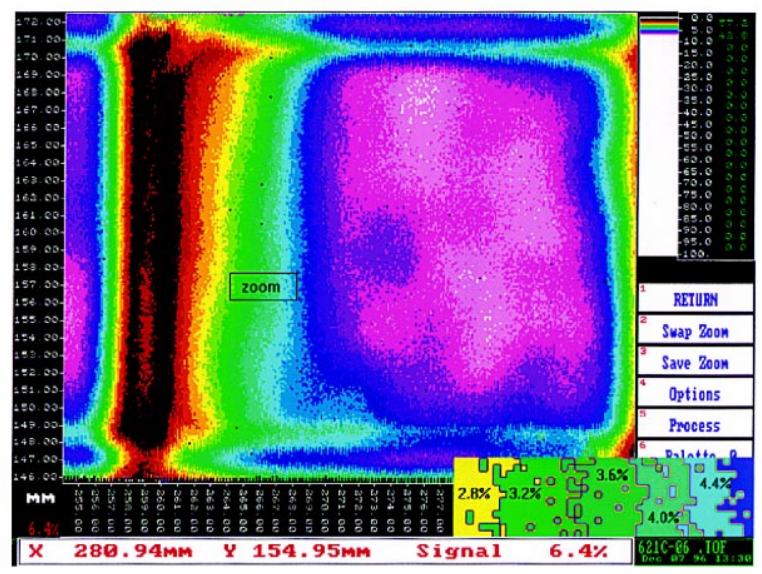

Fig. 3. The crack growth of a woven glass-fabric composite in an interlaminar shear test. (a) elongation $0 \mathrm{~mm}$, (b) elongation $2.167 \mathrm{~mm}$, (c) elongation $2.192 \mathrm{~mm}$, (d) elongation $2.195 \mathrm{~mm}$. 


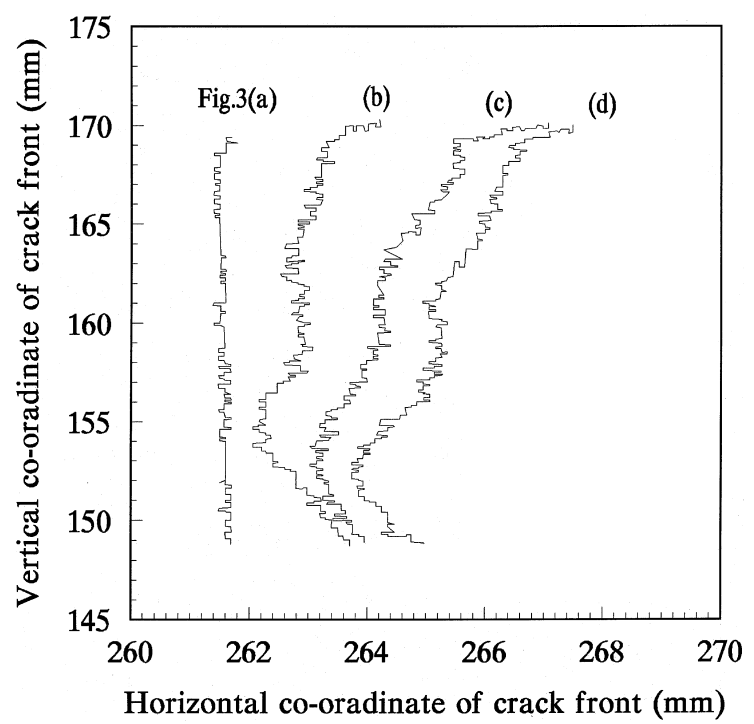

Fig. 4. The damage-growth process obtained by the half-wave method (referring to Fig. 3(a)-(d)).

demonstrate the crack-growth process clearly by comparing the co-ordinates of crack front (Fig. 4). The coordinate of the point at which the crack front intersects the longitudinal axis of the specimen changes with increasing total deformation (Fig. 5). The original crack front is nearly straight. But, with increasing total deformation the shape of the crack front becomes irregular. It seems that cracking is inhomogeneous. The crack propagates slowly when the elongation ranges from zero to $2.167 \mathrm{~mm}$. After that, a tiny increment of total deformation induces a quick propagation of the crack to failure. The specimen fractures completely when the total displacement reaches $2.200 \mathrm{~mm}$.

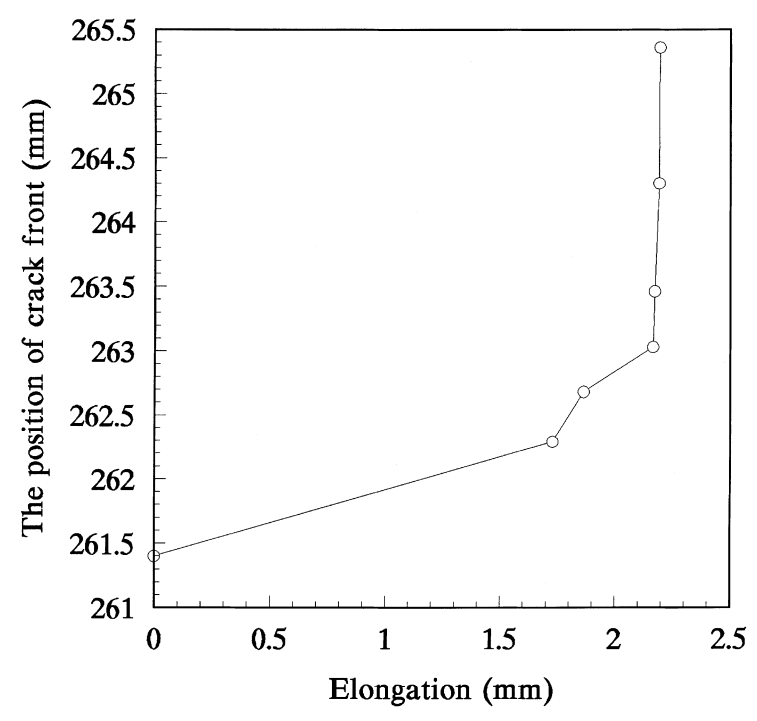

Fig. 5. The curve of crack front and specimen displacement.

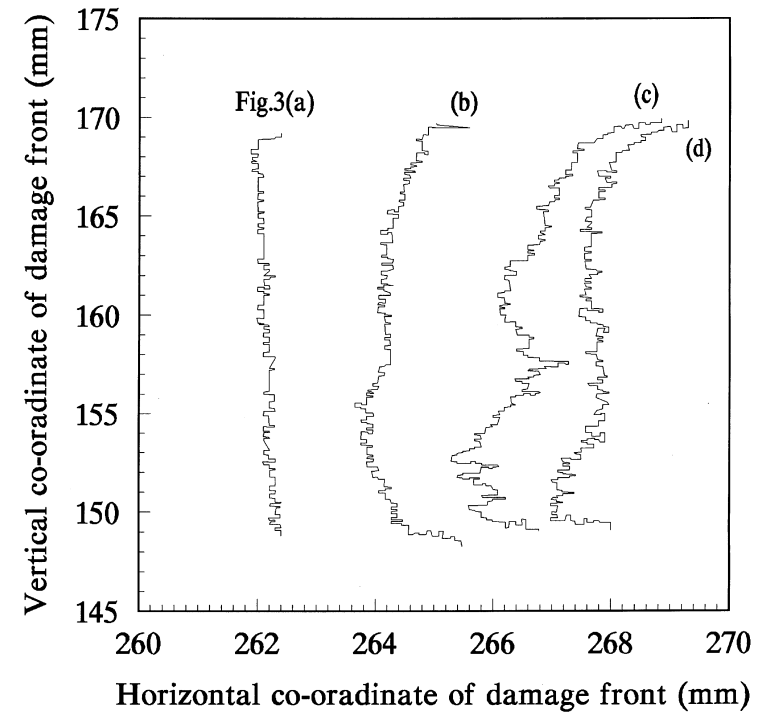

Fig. 6. The damage-growth process obtained according to the region from $3.2 \%$ FSD to $4.0 \%$ FSD (referring to Fig. 3(a)-(d)).

The above data and information are all obtained by the half-wave method. Furthermore, we found a distinct expanding region between the crack front $(2.8-3.2 \%$ FSD) and the intact material (6.4\% FSD). The reduction of the pulse-echo reflection occurring inside the uncracked specimen can only be attributed to a certain damage growth ahead of the crack front. We assumed that the initial edge damage area should be less than $5 \%$ of the whole uncracked specimen. From Fig. 3(a), this corresponds to an RGP amplitude less than 4.4\% FSD. Hence, we define a region whose RGP amplitude is from $3.2 \%$ FSD to $4.0 \%$ FSD as an obvious damage region, and the boundary between $4.0 \%$ FSD and $4.4 \%$ FSD (light blue) is defined as the damage front. Much more importantly, we found that the obvious damage region propagates at a higher speed than the crack front. Fig. 6 shows the damage development process in a way similar to Fig. 4. In fact, the RGP amplitude of this region is higher than a half wave, but is much lower than the maximum amplitude. The above information thus really shows that the material in the region is damaged but not completely fractured. It seems that the woven glass-fabrics begin to separate from the matrix, but do not completely delaminate. Therefore, the boundary between $4.0 \%$ FSD and $4.4 \%$ FSD demonstrates a damage-growth process. This has not been revealed by other methods in interlaminar shear tests.

Comparing Fig. 4 with Fig. 6, it is very clear that both crack and damage fronts propagate with increasing deformation. But the damage area expands more quickly. We can deduce that the propagation of the crack and the rapid propagation of the damage front accompany each other. It may be, then, that the interlaminar 
fracture should be attributed to both crack propagation and more rapid damage extension.

\section{Conclusions}

(1) In order to highlight the damage-growth process under loading, a loading device equipped with an ultrasonic C-scanning test system has been designed. The whole system works very well.

(2) With the loading device, the ultrasonic inspection system successfully records the crack and damagedevelopment process in double-sided grooved composite specimens. The quantitative results show that a small increment of total deformation can induce the rapid propagation of an interlaminar crack in the final stages of damage. This may be due to the more rapidly propagating damage which spreads widely ahead of the crack.

\section{Acknowledgements}

This work was supported by the National Natural Science Foundation of China, under Contract No. 19402018.

\section{References}

[1] Knauss WG. Changes in the experimentalist's role in the future of mechanics. Experimental Mechanics 1996;35:281-92.

[2] $\mathrm{Wu} \mathrm{X}$. A review on experiments of mesomechanics. The Developing Trend of Mechanics of Solid. Beijing University of Technology Press, 1995. p. 216-34 (in Chinese).

[3] Hariharan P. Optical holography. Cambridge: Cambridge University Press, 1984.

[4] Sirohi RS. Speckle metrology. New York: Marcel Dekker, 1993.

[5] $\mathrm{Hu}$ J, Zhang Q. The principle and method of ultrasonic inspection. University of Sciences and Technology of China Press, 1993 (in Chinese).

[6] Posakony J. Ultrasonic inspection. Metals handbook desk edition. ASM, 1985.

[7] Krautkramer J, Krautkramer H. Ultrasonic testing of materials, 2nd ed. Berlin: Springer, 1977.

[8] Bibo GA, Hogg PJ, Backhouse R, Mills A, Deformation mechanics in fabric reinforced composites. In: Proceedings of ICCM-10, vol. IV. Whistler, BC, Canada, 1995. p. 317-24.

[9] Ramanathan S, Kishore. Drop weight repeated impacts and postimpact ilss tests on glass epoxy composites. In: Proceedings of ICCM-10, vol. V. Whistler, BC, Canada, 1995. p. 591-8.

[10] Guo Z. et al. Mechanical design handbook. Sciences and Technology Press of Tianjing, 1988 (in Chinese).

[11] Su Y. Mechanics of materials. Higher Education Press, 1980 (in Chinese).

[12] The Chinese institute of inviolate inspection. Ultrasonic Inspection. The Mechanical Industry Press, 1989 (in Chinese). 